\title{
1 The cold-drought tolerance trade-off in temperate woody plants constrains range 2 size, but not range filling
}

3 Giacomo Puglielli $^{*}$, Enrico Tordoni ${ }^{2}$, Aelys M. Humphreys ${ }^{3,4}$, Jesse M. Kalwij ${ }^{5,6}$, Michael J.

$4 \quad$ Hutchings ${ }^{7}$, Lauri Laanisto ${ }^{1}$

$5{ }^{1}$ Institute of Agricultural and Environmental Sciences, Estonian University of Life Sciences,

6 Tartu, 51006, Estonia;

$7{ }^{2}$ Institute of Ecology and Earth Sciences, University of Tartu, Lai 40, 51005 Tartu, Estonia;

$8{ }^{3}$ Department of Ecology, Environment and Plant Sciences, Stockholm University, SE-106 91

9 Stockholm, Sweden;

$10{ }^{4}$ Bolin Centre for Climate Research, Stockholm University, SE-106 91 Stockholm, Sweden;

$11{ }^{5}$ Institute of Geography and Geoecology, Karlsruhe Institute of Technology, Karlsruhe,

12 Germany;

$13{ }^{6}$ Centre for Ecological Genomics and Wildlife Conservation, Department of Zoology, University

14 of Johannesburg, Auckland Park 2006, South Africa;

$15{ }^{7}$ School of Life Sciences, University of Sussex, Falmer, Brighton, Sussex, BN1 9QG, United

16 Kingdom.

17 * Author for correspondence - Email: Giacomo.Puglielli@emu.ee

18 Acknowledgements: The Estonian University of Life Sciences provided funding for this work

19 (grant number: P200187PKEL and P200190PKEL awarded to GP and LL, respectively). GP also

20 thanks Babak Naimi for helpful discussion on the implementation of SDMs. 


\section{Abstract}

Interspecific differences in plant species' ranges are shaped by complex mechanistic interactions, which have so far remained largely beyond the reach of comprehensive models and explanations. Previous attempts to find underlying mechanisms by examining physiological tolerances to cold and heat separately have yielded contradictory results. Here we test the hypothesis that, instead of examining single stressors, abiotic stress tolerance syndromes that involve trade-offs between multiple abiotic stressors (namely drought, cold, waterlogging and shade), will provide reliable explanations.

We compiled a dataset of actual range size and range filling (the ratio between actual and potential species range) as range metrics for 331 temperate woody plants species from Europe and North America. Tolerance syndromes were expressed as two PCA axes. One axis reflects a drought-cold/waterlogging tolerance trade-off (cold/wet-drought trade-off), the second axis represents a shade tolerance spectrum. Phylogenetic generalized linear mixed models were used to model the range metric-tolerance axes relationships using latitude as an additional main effect, and phylogeny and plant functional type as random effects.

Actual range scaled negatively with the cold/wet-drought tolerance trade-off axis, mostly independently of latitude and continent. Thus, cold/wet-tolerant species had the largest ranges and drought tolerant species the smallest. The sign (-) of the relationship was independent of phylogeny and plant functional type. In contrast, range filling depended on latitude. However, deciduous and evergreen species displayed different distributions of range metrics and tolerance syndromes. No significant relationships with the shade tolerance spectrum were found.

Our findings demonstrate that the cold/wet-drought trade-off partly explains interspecific range

50 size differences. However, this trade-off did not explain range filling. We also showed that

51 fundamental adaptations of species also significantly influence range sizes - stress avoidance

52 through the deciduous habit also explained interspecific differences in range size.

54 Keywords: abiotic stress, plant species distributions, cold tolerance, drought tolerance,

55 Hutchinsonian niche, range filling, range size, woody plant species 


\section{Introduction}

57 The identification of the factors shaping species range size and filling is a major focus of biogeography (Brown, 1984; Gaston et al., 2009). In recent years, a number of theories aimed at explaining species distribution patterns at the global scale have been developed. For example, the mid-domain effect predicts that species close to the equator have larger distribution ranges (Colwell \& Hurtt, 1994), whereas Rapoport's rule assumes the opposite (Stevens, 1989). Several mechanistic hypotheses either addressing extrinsic factors (e.g. the climate variability hypothesis (Stevens, 1989) to explain Rapoport's rule) or intrinsic factors (e.g. the dispersal (Hanski et al. 1993) or niche breadth (Brown, 1984) hypotheses) have been proposed (reviewed in Sheth et al., 2020). Most of the hypotheses formulated so far to explain interspecific differences in range size have emerged from zoology (Fine, 2015). In comparison, this topic has been little investigated in plant species (Sheth et al., 2020), for which the determinants of range size, and its global variation, remain elusive.

Different metrics of range size are used in the literature to describe plant biogeographic patterns (Sheth et al., 2020). These metrics include latitudinal or longitudinal range, extent of occurrence or area of occupancy, along with the ratio of realized range size (based on known species distributions) over potential range size (usually estimated using species distribution models), also known as range filling (Svenning \& Skov, 2004; Paul et al., 2009; Sheth et al., 2020). Despite the use of different metrics, some consistent biogeographic patterns have emerged. For example, North American regions with strong climate instability have lower species richness and large-ranged species, while small-ranged species inhabit more species-rich regions with more stable climates (Morueta $\square$ Holme et al., 2013; McFadden et al., 2019). For European tree species, both potential range size and range filling increase with latitude (Svenning \& Skov, 2004; Nogués-Bravo et al., 2014), and both European and North American woody species tend to have larger ranges at higher latitudes (Morin \& Chuine, 2006).

All these results are consistent with Rapoport's rule, which predicts larger ranges and lower species richness at higher latitudes, possibly due to high latitude species being more

83 tolerant of more variable environmental conditions (e.g., Morueta $\square$ Holme et al., 2013), or to an 84 increased frequency of pioneer species with broader niches at higher latitudes (Morin \& Chuine, 2006). Despite these general tendencies, exceptions to Rapoport's rule have been documented. 
For example, in the Americas, woody species' range size has a bimodal distribution in relation to latitude, being largest in both north temperate and tropical areas (Weiser et al., 2007). A similar bimodal distribution has been observed for range filling in European (Svenning \& Skov, 2004) and North American tree species spanning sub-tropical to boreal climates (Seliger et al., 2021). Thus, although a positive range size-latitude relationship is well documented for plants, it is not without exceptions and a mechanistic explanation of this pattern is still missing.

Morin \& Chuine (2006) proposed that the proximate driver behind Rapoport's rule is abiotic stress tolerance. In particular, they argued that intrinsic differences in abiotic stress tolerance between species, and thus in their ability to persist under given resource regimes, might explain interspecific variation in range size. Following this proposal, analysis of species' inherent abilities to withstand extreme heat and/or cold has provided the main way to seek a link between species' physiology and distribution ranges, chiefly expressed as latitudinal limits (mostly for animals, e.g. Addo-Bediako et al., 2000; Gaston et al., 2009; Sunday et al., 2011; Araújo et al., 2013). Comprehensive large-scale datasets of thermal tolerances have only appeared recently for plant species (e.g., Lancaster \& Humphreys, 2020). Nevertheless, relating species' abiotic stress tolerances to their distribution ranges is complex. Cold and heat tolerance, for instance, only have clear relationships with latitudinal and climatic gradients under certain conditions. Cold tolerance seems to be more closely related to climatic conditions than heat

105

106 tolerance (Araújo et al., 2013; Lancaster \& Humphreys, 2020), and patterns are stronger for northern hemisphere than southern hemisphere species. Similarly, cold and drought tolerances do not display straightforward relationships with range filling, despite showing a clear latitudinal pattern across Europe (Nogués-Bravo et al., 2014). Thus, our comprehension of the relationship between abiotic stress tolerance and species distributions remains poor.

Relating species' physiological tolerances to their geographical distribution patterns is complicated by occupied ranges representing realized niches (e.g. Hutchinson, 1957), whereas physiological tolerances should reflect species' fundamental niches. Furthermore, physiological tolerances of different stressors might trade-off against each other due to correlation with independent niche axes of the Hutchinsonian hypervolume (Sexton et al., 2017), shrinking the number of feasible tolerance combinations. Accounting for trade-offs between multiple tolerances may more closely reflect species' realized physiological requirements (Sack, 2004; 
Niinemets \& Valladares, 2006; Laanisto \& Niinemets, 2015; Puglielli et al., 2021a) and possibly reveal consistent relationships with realized range sizes. Thus, multivariate trade-off axes between different tolerances might be needed to detect correlates with range size. Recently, Puglielli et al. (2021 a) examined multivariate trade-offs in woody species' ecophysiological tolerances of four major abiotic stresses (cold, shade, drought and waterlogging). The trade-offs were visualized in a triangular stress tolerance space (henceforth Stress Space). Ecophysiological tolerance is defined as a species' ability to survive long-term extreme shortage of a given resource in its natural environment (Niinemets \& Valladares, 2006). The Stress Space was built using published species-specific tolerance data (Niinemets \& Valladares, 2006; Laanisto \& Niinemets, 2015) for $\sim 800$ Northern Hemisphere woody plant species. Each pair of coordinates in the Stress Space reflects species-specific multi-stress tolerance syndromes shaped by tradeoffs among the different tolerances. In particular, the first Stress Space axis reflects a trade-off between drought and cold/waterlogging tolerance. The second axis is a shade tolerance spectrum, from low- to high shade tolerance. Thus, the Stress Space framework permits to link abiotic stress tolerance syndromes to other aspects of species' biology, including range size. A welldeveloped framework describing the relationship, and potential trade-offs, between multiple abiotic stress tolerances is crucial for making realistic inferences about the role of abiotic stress tolerance in shaping species distribution patterns, including global variation in range sizes (Gaston et al., 2009).

Identification and interpretation of range size correlates has the potential to increase understanding of the factors and processes that influence species' ranges (Svenning \& Skov, 2004; Estrada et al., 2016). Although a complete spectrum of range size correlates is not yet available (Estrada et al., 2016, 2018), especially for plants, we employ a dataset on 300 temperate woody species from Europe and North America, to analyze the relationships between abiotic stress tolerance syndromes, as summarized by the Stress Space axes, and species actual range sizes and range filling. We decided to consider both of these range metrics because they reflect different aspects of species' ranges. Actual range size includes historical legacies (e.g. for temperate species, the degree to which they have been able to expand since the Last Glacial Maximum; Svenning et al., 2008; Normand et al., 2011; Nogués-Bravo et al., 2014; Estrada et al., 2016) and ecological constraints (e.g. areas with suitable habitat, Linder et al., 2013). Range filling, on the other hand, is a measure of the extent to which species' ranges are at a climatic 
147 equilibrium (Svenning \& Skov, 2004). Thus, the traits that are positively correlated with actual

148 range size are expected to be those that are associated with range expansion (Estrada $e t$ al.,

149 2016, 2018), whereas traits associated with range filling reflect limits on species' distributions

150 imposed by historical non-climatic factors (Estrada et al., 2016). Previous studies have mostly

151 attempted to link range filling to plant dispersal syndromes (Svenning et al., 2008; Normand et

152 al., 2011; Nogués-Bravo et al., 2014). However, even assuming successful migration and

153 dispersal, the regeneration niche theory (Grubb, 1977), also requires that a species is able to

154 survive the prevailing abiotic (and biotic) conditions to establish viable populations (Estrada et

155 al., 2018). The Stress Space framework can therefore provide further insights into the

156 determinants of species' range size and range filling.

In this study, we examined the relationships between temperate woody species range sizes and range filling, and the Stress Space axes. Assuming these metrics are positively correlated (e.g. Seliger et al., 2021), we propose that species' abiotic stress tolerance syndromes (reflected in their positioning along the Stress Space axes) can largely explain latitudinal differences in range size and filling. Specifically, we hypothesized that:

1) As the first Stress Space axis represents a spectrum from cold/waterlogging- to drought tolerant species, and assuming this reflects a latitudinal gradient (Nogués-Bravo et al., 2014), we expected a negative relationship between both range metrics and this axis.

2) Both range metrics will be independent of shade tolerance, which in turn is independent of latitude. Shade tolerance data used in the Stress Space are measures of a species' capacity for growth in low light conditions compared to the capacity for growth of coexisting species (Niinemets \& Valladares, 2006). Shade tolerance is also largely independent of the other tolerances in the Stress Space (Puglielli et al., 2021a). Therefore, shade tolerance can be either high or low irrespective of latitude. 


\section{Methods}

\section{Actual range and occurrence data}

We carried out an extensive literature search for polygons defining species' actual ranges for the 799 woody species in Puglielli et al. (2021a). We were able to retrieve polygons defining species' actual range for 331 species. Specifically, spatial distributions of North American species $(n=201)$ were obtained from the "Digital representations of tree species range maps from Atlas of United States Trees" (available at https://github.com/wpetry/USTreeAtlas). Distributions of European species $(n=130)$ were gathered from the International Union for Conservation of Nature (IUCN, www.iucnredlist.org), the European forest genetic resources program (EUFORGEN, http://www.euforgen.org/species), and published papers (Kalwij et al., 2014; Caudullo et al., 2017; Wazen et al., 2020).

Species occurrence records for the 331 species were obtained from the Global Biodiversity Information Facility (GBIF, www.gbif.org/, accessed 21/12/2018; full list of data sources in Appendix S1, Supporting Information Table S1.1). GBIF data were carefully cleaned using both standardized and customized procedures (see Appendix S1, Fig. S1.1).

\section{Potential range size and range filling calculation}

To estimate species' potential range sizes, we used two presence-only models - i.e., Bioclim (Busby, 1986), and Maxent (Phillips et al., 2006) - to account for differences in potential range size that may arise from algorithmic differences ( Nogués-Bravo et al., 2014). Each model was fitted with three environmental parameters: growing degree days at $5^{\circ} \mathrm{C}$ (GDD, unitless); climatic moisture index (the ratio of annual precipitation to annual potential evapotranspiration, CMI, unitless) and mean minimum temperature of the coldest month $\left(\mathrm{T}_{\min },{ }^{\circ} \mathrm{C}\right)$. GDD and CMI data were obtained from the ENVIREM dataset (Title \& Bemmels, 2018) and $\mathrm{T}_{\min }$ (i.e. Bio6) from WorldClim (Hijmans et al., 2005); each at 10 arcmin resolution.

Before computing Species Distribution Models (SDMs), occurrence data were subjected to environmental filtering following Varela et al. (2014; see Appendix S1, Fig. S1.1). All the 331 species had $>20$ occurrences, which is considered a reasonable threshold for fitting SDMs (Guisan et al., 2017).

The SDMs were fitted using the $s d m$ R package (Naimi \& Araújo, 2016). For each run, $80 \%$ of species data was used for training, and the remaining $20 \%$ for evaluating the model. 30 
replicates per species were generated through bootstrapping and 20,000 background points were generated at each run. The Area under the ROC Curve (AUC; Fielding \& Bell, 1997) and True Skill Statistic (TSS; Allouche et al., 2006) were used to evaluate model performance. The SDMs predictions were converted into presence/absence maps by using the threshold that maximized both sensitivity and specificity of the model. This is considered the best option for presence-only methods (Liu et al., 2013). The number of suitable 10 arcmin cells in the binary maps corresponded to potential range size while actual range was determined by counting the 10 arcmin cells occupied by the polygons defining species' actual range. Range filling (\%) was then calculated as: (Actual range/Potential range)×100 (see Appendix S2, Fig. S2.2). Due to a lower percentage of species with range filling estimates greater than 100\% (see Appendix S3, Figs.

S3.3-3.6, Table S3.2 for considerations on models' performance and range filling estimates) only Bioclim derived estimates of potential range were used in subsequent analyses.

In order to account for broad differences in species' adaptive syndromes, species were classified according to three major plant functional types: deciduous angiosperms, evergreen angiosperms and evergreen gymnosperms. For the complete list of species' actual range size ( $\log _{10}$-transformed number of 10 arcmin cells), range filling, centroid latitude and species classification according to their continental origin (N. America, Europe) and plant functional type see Appendix S4.

\section{Abiotic stress tolerance data}

The species-specific estimates of tolerance of shade, drought, cold and waterlogging used to define the Stress Space were obtained from the datasets of Niinemets \& Valladares (2006) and Laanisto \& Niinemets (2015), which include stress tolerance scores for $~ 800$ Northern Hemisphere woody species. In the original data compilation (Niinemets \& Valladares, 2006), shade, drought and waterlogging tolerance were independently estimated by cross-calibrating multiple tolerance scales reported in the literature where multiple measurements for one species were available across tolerance scales. Cold tolerance data were extracted from USDA plant hardiness data (Laanisto \& Niinemets, 2015). All the stress tolerance scores were then harmonized to fit a 5-level scale (1 - very intolerant; 5 - very tolerant) (Niinemets \& Valladares 2006 and Laanisto \& Niinemets 2015). 

dimensions (principal components) capture $\sim 80 \%$ of the variance in species-specific combinations of shade, drought, cold and waterlogging. Each pair of coordinates in the Stress Space corresponds to a species-specific stress tolerance syndrome. Stress Axis 1 is positively

236 correlated with drought tolerance and negatively correlated with both waterlogging and cold

237 tolerance. It is interpreted here as a cold-drought tolerance trade-off, where the term cold refers

238 to a short growing season. This interpretation stems from the positive covariance between cold

239 and waterlogging tolerance in our dataset: the highest cold tolerance is expected where snowpacks are greater, resulting in later snowmelt, followed by waterlogging and consequently a shorter growing season (Chuine, 2010). Stress Axis 2 is positively correlated with shade

242 tolerance, and represents a shade tolerance spectrum. Stress Axes are available in Puglielli et al. 243 (2021a).

\section{Data analysis}

245 Actual range size (hereafter range size) was log-transformed before analysis. Range filling was

246 strongly related with range size $\left(R^{2}=0.46, \mathrm{p}<0.0001, n=331\right)$, but not with $\log _{10}$-transformed 247 potential range size $\left(R^{2}=0.02, \mathrm{p}<0.001, n=331\right)$. Thus, we used the residuals deriving from 248 the relationship range size vs. range filling as a metric of range filling (Seliger et al., 2021).

The relationships between stress axes and both range size and range filling residuals were tested at different levels. First, we used Ordinary Least Square (OLS) regression analysis. Second, we used quantile regression to provide a more comprehensive characterization of the studied relationship (Ricotta et al., 2010). In addition, quantile regression is mostly insensitive to

253 outliers (Ricotta et al., 2010). Quantile regressions were run using the quantreg R package

254 (Koenker, 2017) using different quantiles of range metrics distribution $(\tau=0.1, \tau=0.25, \tau=$ $2550.50, \tau=0.75$ and $\tau=0.90)$. However, fitting models with many species while ignoring 256 phylogenetic relationships might lead to inflated type I errors (Freckleton et al., 2002).

257 Consequently, as a third step, we computed Phylogenetic Generalized Linear Mixed Models 258 (PGLMMs, Ives \& Helmus, 2011) using range metrics as response variable as a function of 259 stress axis 1 or 2 (considered separately), latitude (centroid latitude calculated using the 260 geosphere R package, Hijmans et al., 2019) plus the first-order interaction stress axis : latitude; 261 species' phylogenetic relatedness and plant functional types were considered as random effects. 
262

263

264

265

266

267

268

269

270

271

272

273

274

275

276

277

278

279

280

281

282

283

284

285

286

287

288

289

290

When the interaction was not found to be significant, only the main effects were considered. PGLMMs were run using the phyr $\mathrm{R}$ package (Li et al., 2020). Phylogenetic data were retrieved for 325 species in our dataset using the mega-tree available via the V.PhyloMaker R package (Jin \& Qian, 2019). The mega-tree combines the phylogenies developed by Zanne et al. (2014) and Smith \& Brown (2018). Species nomenclature followed The Plant List v.1.1 (2013). We also tested for potential signals of spatial autocorrelation in the model residuals using spline correlograms from the $n c f$ R package (Bjørnstad, 2020); specifically, 95\% pointwise bootstrap confidence intervals were computed from 5,000 bootstrap samples of Pearson residuals.

Finally, we tested for differences between plant functional types in terms of the distributions of actual range and range filling residuals, and positioning along stress axes using the Kruskal-Wallis test. Pairwise multiple comparisons between group levels were carried using Dunn's test by adjusting p-values with Holm correction.

All the data analysis procedures were repeated for the European and North American species separately to account for possible geographic differences in patterns observed. All statistical analyses were performed in R 4.0.5 (R Core Team, 2021). As we did not find any relationship between either range size or range filling residuals with the shade tolerance spectrum at any level of analysis, only the results relative to the cold-drought tolerance trade-off axis are shown.

\section{Results}

Actual range scaled negatively with species positioning along the cold-drought tolerance tradeoff axis for species from both continents (Europe: slope $=-0.10, R^{2}=0.06, p=0.01, n=130$; North America: slope $\left.=-0.18, R^{2}=0.14, p<0.01, n=201\right)($ Fig. 1 a,c). A negative relationship between actual range and cold-drought tolerance trade-off axis was also observed across the considered quantiles of the response variable, but with differences between the species from the two continents. For European species, the quantile regressions were mostly significant at average to high values of actual range (Fig. 1 a). For North American species quantile regressions were all significant, except the one fitted at the lowest quantile (Fig. 1 c).

Range filling residuals scaled negatively with species positioning along the cold-drought tolerance trade-off axis only for European species ( 
and, as for actual range, the quantile regressions were mostly significant at average to high values of range filling (Fig. 1 b). No significant relationship was found between range filling residuals and the cold-drought tolerance trade-off axis for North American species, and the relationship was not significant at any considered quantile (Fig. 1 d).

(a) Europe

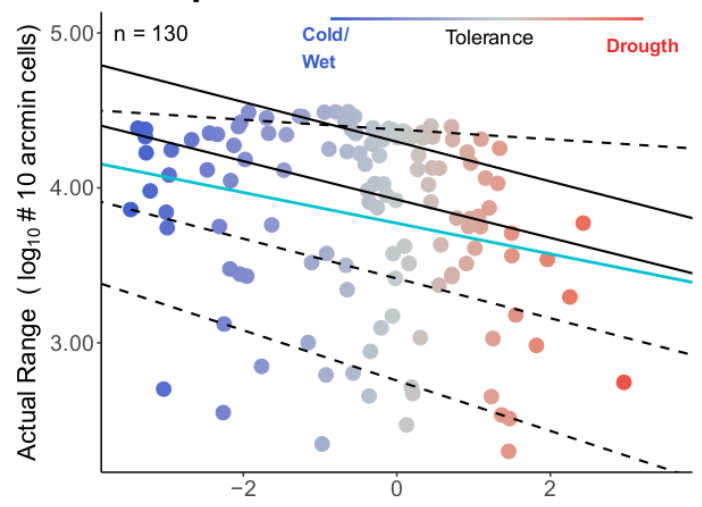

(c) North America

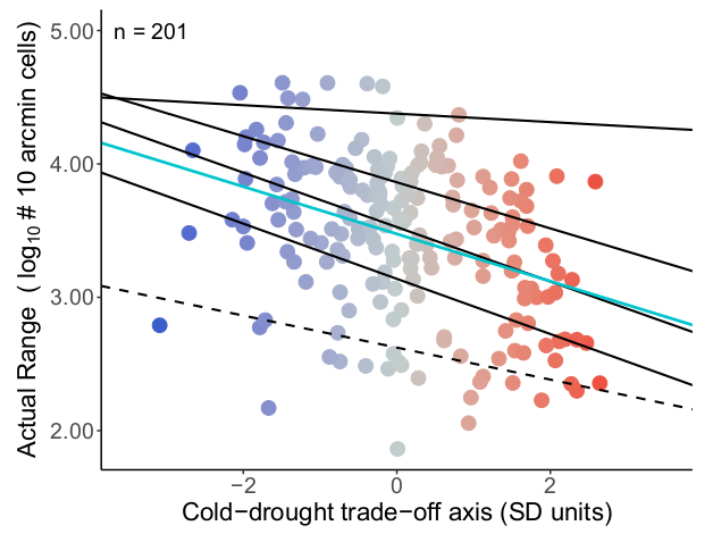

(b)

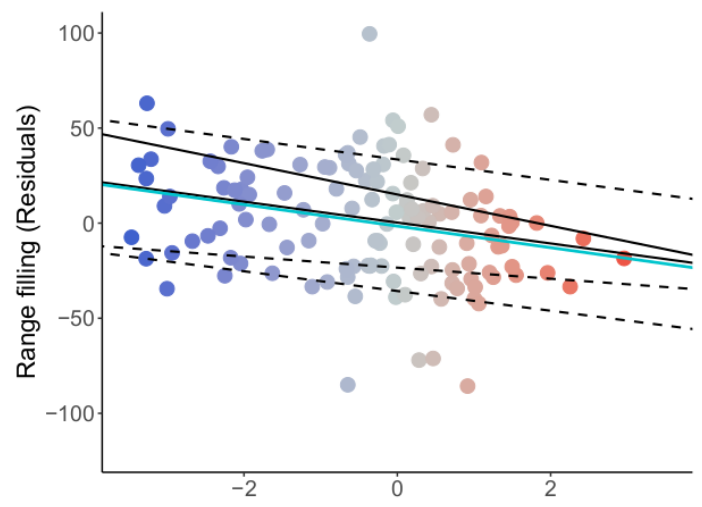

(d)

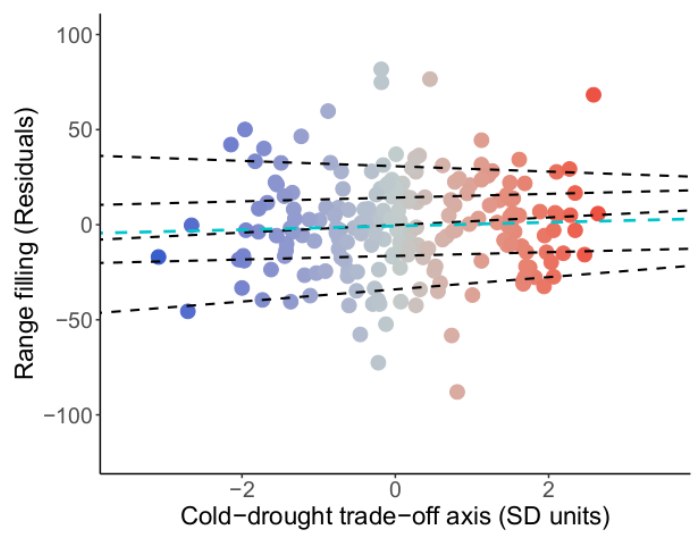

Fig. 1. Relationship between the cold-drought trade-off (Standard Deviation units) and actual range ( $\log _{10}$-transformed number of 10 arcmin cells) and range filling (residuals, see Methods) for: (a,b) European, and (c, d) North American woody plant species. Pale blue line represents the Ordinary Least Square fit. Black lines show the quantile regressions fitted at the $0.1,0.25,0.50,0.75$ and 0.90 quantile of the response variable distribution. Solid lines depict significant relationships at $p<0.05$ while dashed lines represented not significant relationships. Sample size (n) is shown in panels (a,c) and applies also to the relationships involving range filling as the response variable. The color gradient reflects the progression from cold/wet-tolerant to dry/warm-tolerant species along the cold-drought trade-off.

The negative relationship between actual range and the cold-drought tolerance trade-off axis was not affected by including latitude as an additional main effect (Table 1), and the colddrought tolerance trade-off axis effects were always greater than that of latitude for all data pooled (see Appendix S5, Table S5.3), and for each continent considered separately (Table 1). 
However, some differences between continents were detected. The cold-drought tolerance tradeoff axis effect was only marginally significant $(\mathrm{p}=0.06)$ for European species and the effect of latitude was not significant. The model explained $7 \%$ of the variance of actual range for European species. In contrast, for North American species, the effects of both the cold-drought tolerance trade-off axis and latitude were significant, but with opposite sign: the tolerance axis maintained its negative relationship with actual size whereas latitude showed a positive relationship ( $\mathrm{p}<0.01$ and $\mathrm{p}<0.05$, respectively). This model explained $23 \%$ of the total variation in actual range of North American species, and indicates that range sizes are greatest at higher levels of cold/wet tolerance and at higher latitudes. Despite European and North American species sets having no species in common, we explored whether differences between continental species sets could be driven by differences in terms of genera composition.

Out of 106 genera in our entire dataset, there were 29 genera in common between the two continents. These genera contributed $70 \%$ of the species included in the entire dataset. When the analyses were repeated after removing genera that were unique to one or other continent, the result remained significant for North American species, but the relationship between range size and the cold-drought tolerance trade-off axis changed from being marginally significant to become highly significant for European species as well $\left(\mathrm{R}^{2}=0.12\right.$, see Appendix S5, Table S5.4). This indicates that differences in results between continents are partly driven by differences in genera composition of their constituent species.

There was no significant interaction between the cold-drought tolerance trade-off axis and latitude in any model involving actual range as the response variable for European or North American species. Overall, species positioning along Stress Axis 1 was the main driver of interspecific differences in actual range for both continents. Actual range data in relation to the cold-drought tolerance trade-off axis and latitude are shown in Appendix S5, Fig. S5.7a,c.

The differences in results between continents were more pronounced for range filling (Table 1). A significant positive interaction between the cold-drought tolerance trade-off axis and Latitude was found in the model including range filling for European species, and it explained approximately $19 \%$ of the variance. Despite a significant main effect of the colddrought tolerance trade-off axis on range filling (Table 1), we did not interpret this effect given the presence of a significant interaction. We regard the interaction between the cold-drought 
338 tolerance trade-off axis and latitude as the main driver of interspecific differences in range filling

339 across European species. For North American species, range filling showed a negative

340 relationship with latitude and no significant effect of the cold-drought tolerance trade-off axis.

341 This model suggests greater range filling at lower latitudes, but it explained only $4 \%$ of variance,

342 leaving range filling largely unexplained for North American species. Range filling data in

343 relation to the cold-drought tolerance trade-off axis and latitude are shown in Appendix S5, Fig.

344 S5.7b,d.

345

Regardless of the model, the effect of phylogenetic relatedness between species was

346 negligible (Table 1). In addition, the spline correlograms (see Appendix S6, Fig. S6.8) did not

347 reveal any evidence of spatial autocorrelation in the PGLMMs residuals. We can therefore safely

348 disregard spatial autocorrelation as a factor influencing model parameter estimates. Plant

349 functional types did not affect the strength or the sign of the relationships between the cold-

350 drought tolerance trade-off axis and either actual range or range filling. However, as a random

351 effect in PGLMMs, plant functional type had a greater effect overall than species phylogenetic

352 relatedness in terms of random effect variance (Table 1). 


\begin{tabular}{|c|c|c|c|c|c|c|c|c|c|}
\hline \multirow[b]{2}{*}{ Response } & \multirow[b]{2}{*}{ Continent } & \multicolumn{6}{|l|}{ Main effects } & \multicolumn{2}{|c|}{ Random effects } \\
\hline & & & Estimate & $\mathbf{S E}$ & $p$ & Model $R^{2}$ & $\mathbf{n}$ & & Variance \\
\hline \multirow[t]{6}{*}{ Actual range } & Europe & Intercept & 3.39 & 0.34 & $<0.0001$ & 0.07 & 128 & PFT & 0.007 \\
\hline & & Cold-drought trade-off & -0.07 & 0.04 & 0.06 & & & Phylogeny & $\approx 0$ \\
\hline & & Latitude & 0.01 & 0.01 & $\mathrm{~ns}$ & & & & \\
\hline & North America & Intercept & 2.9 & 0.22 & $<0.0001$ & 0.23 & 197 & PFT & 0.05 \\
\hline & & Cold-drought trade-off & -0.11 & 0.03 & $<0.001$ & & & Phylogeny & $\approx 0$ \\
\hline & & Latitude & 0.01 & 0.01 & $<0.01$ & & & & \\
\hline \multirow[t]{7}{*}{ Range filling } & Europe & Intercept & 27.08 & 17.37 & ns & 0.19 & 128 & PFT & 33.12 \\
\hline & & Cold-drought trade-off & -43.18 & 11.77 & $<0.0001$ & & & Phylogeny & 0.003 \\
\hline & & Latitude & -0.56 & 0.34 & ns & & & & \\
\hline & & Cold-drought trade-off $*$ Latitude & 0.69 & 0.22 & $<0.001$ & & & & \\
\hline & North America & Intercept & 22.96 & 9.25 & 0.05 & 0.04 & 197 & PFT & 3.97 \\
\hline & & Cold-drought trade-off & 0.01 & 1.56 & ns & & & Phylogeny & 0.001 \\
\hline & & Latitude & -0.6 & 0.22 & $<0.001$ & & & & \\
\hline
\end{tabular}

361 Models were run by continent (Europe and North America) using actual range (log10-transformed number of 10 arcmin cells) and range filling (Residuals, see Methods) as

362 response variables, Cold-drought trade-off (Standard Deviation units) and latitude (centroid latitude, ${ }^{\circ}$ ) as main effects, and Plant Functional Type (PFT) and species

363 phylogenetic relatedness (Phylogeny) as random effects. Estimates of the main effects and their standard errors (SE), together with the variance explained by the model

364 (Model $R^{2}$ ) and sample size (n), are shown. The variance captured by the random effects is also shown. 
The three plant functional types differed in distributions of actual range and range filling values and in positioning along the cold-drought tolerance trade-off axis (Kruskal-Wallis test, $\mathrm{p}$ $\leq 0.05$; Fig. 2 a-f). As a general trend, deciduous angiosperms have larger actual range and range filling values ( $\mathrm{p} \leq 0.05$; Fig. 2 a,b,d,e), and they occupy the cold/wet side of the cold-drought tolerance trade-off (i.e. more negative values along the cold-drought tolerance trade-off axis) (Fig. 2 c,f), compared to the other plant functional types. However, multiple comparisons sometimes differed between continents (Fig. 2 a-f).
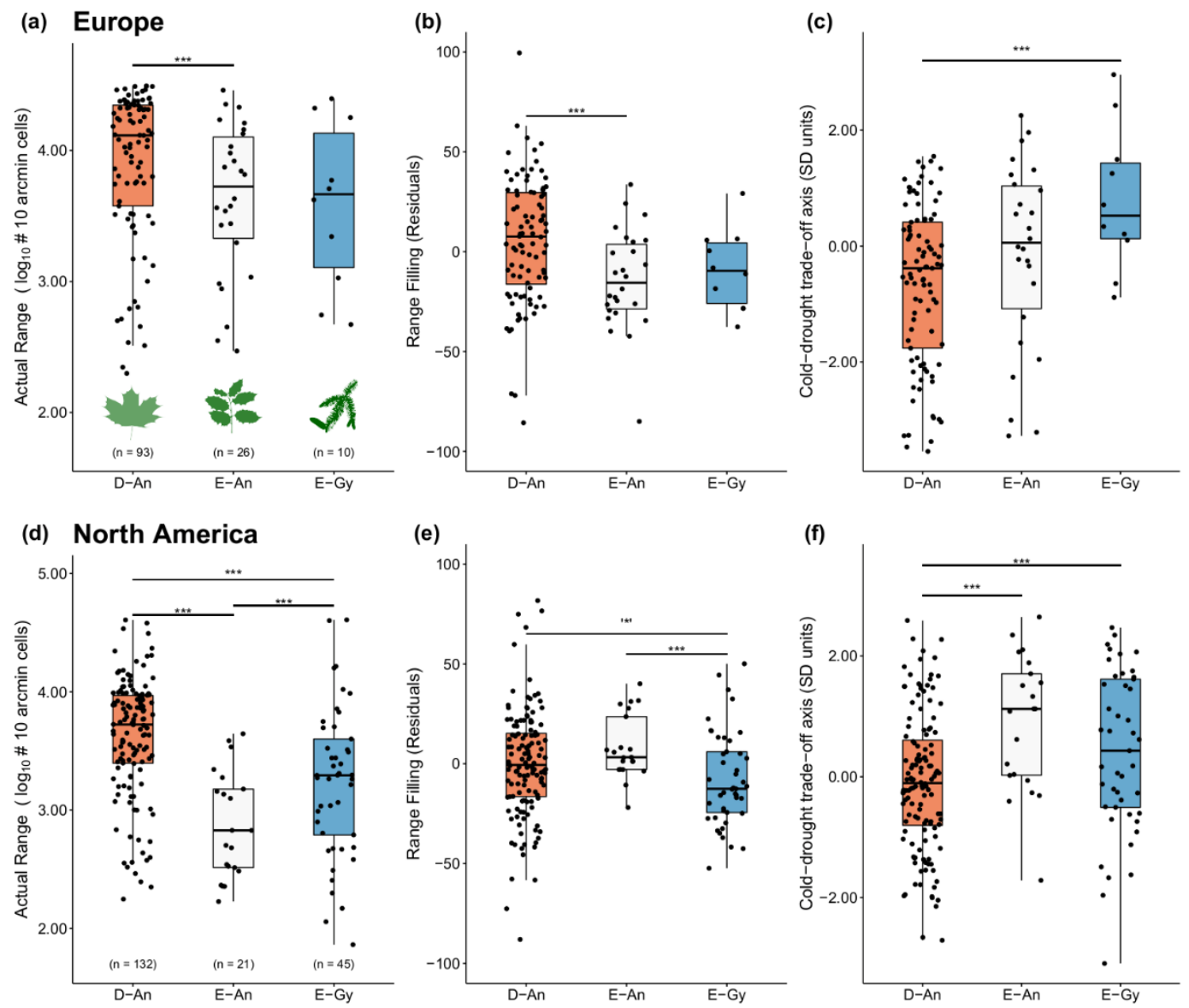

Fig. 2. Boxplots for the distribution of actual range size ( $\log _{10}$-transformed number of 10 arcmin cells), range filling (Residuals, see Methods) and positioning of species along the cold-drought trade-off (Standard Deviation units) within each of the plant functional types (D-An = deciduous angiosperms; EAn = evergreen angiosperms; E-Gy = evergreen gymnosperms) for: (a-c) European, and (d-f) North American woody species. Sample size and data points for each plant functional type are shown. *** indicates significant differences between plant functional types (Kruskal-Wallis, $p<0.05$ ). ' $*$ ' indicates marginal significance $(\mathrm{p}=0.05)$. Only significant and marginally significant differences are shown. Multiple comparisons between groups were carried out using the Dunn's test and Holm correction for multiple testing. 


\section{Discussion}

Our results show that the cold-drought tolerance trade-off axis (hereafter cold-drought trade-off) constrains actual range size of temperate woody species (Fig. 1). In particular, we found largeranged species at the cold/wet tolerance end of the trade-off axis, and small-ranged species at the drought tolerance end. Despite some nuanced variation discussed below, this result was independent of continent, latitude, plant functional type and phylogeny, indicating its generality. In contrast, range filling showed different patterns in Europe and North America: the interaction between the cold-drought trade-off and latitude had the largest influence on range filling for European species, whereas latitude was the only driver of range filling in our North American species pool.

\section{Determinants of range size variation in temperate woody plants}

Occurrence in cold, highly seasonal environments is associated with large ranges in trees (Stevens, 1989; Pither, 2003; Morin \& Chuine, 2006; Morueta $\square$ Holme et al., 2013). Hypotheses to explain this include the climate variability hypothesis (Stevens, 1989), the niche breadth hypothesis (Brown, 1984), and the likelihood of cold-tolerant species having had northerly refugia prior to the Last Glacial Maximum, enabling faster colonization of newly available habitat after ice retreat (Svenning et al., 2008).

For North American species, we found that cold/wet tolerant species occurring at high latitudes (e.g. Salix spp., Larix laricina (Du Roi) K.Koch) generally have the largest ranges (Table 1). Consistent with this, large ranged, cold-tolerant North American tree species are known to be generally absent from regions that are consistently warm and moist, such as the southeastern regions of the continent (Pither, 2003). Palaeoecological records also provide evidence for rapid northward range shifts in North American large-ranged trees after the latest ice age (Seliger et al., 2021, and references herein). Conversely, some species might have maintained relatively small realized ranges following deglaciation, perhaps due to trait syndromes guaranteeing competitive advantages only in specific ice age refugia (Seliger et al., 2021). Possible examples are species with drought tolerance strategies that prevented northward range expansions after glacial retreat, such as Juniperus deppeana Steud., Pinus monophylla Torr. \& Frém., and Quercus douglasii Hook. \& Arn., among other species with relatively small ranges that are confined to SW-North America. Long-term drought, and likely adaptations to 
tolerate such conditions, are an important constraint on plant species distributions (e.g. Normand et al., 2009). Similarly, Pither (2003) hypothesized that latitudinal patterns in the range sizes of

North America woody species reflect a potential trade-off between species' cold tolerance strategies and their competitiveness in warmer environments. In support of this, our results show that the cold-drought trade-off, which largely reflect species biogeographical history, is a mechanism that actively influences interspecific differences in range size for North American plant species.

Cold-tolerant European species also have large ranges (Fig. 1 a), but the signal was weaker than for North American species (Table 1). This difference is caused by differences in genera composition between the continental species sets, and by a cluster of European species with intermediate positions along the cold-drought trade-off having large ranges (Fig. 1 a). These large-ranged species include Picea abies (L.) H.Karst, Pinus sylvestris L. and Betula pendula. Roth. Such species survived the last glacial maximum in central and/or eastern Europe with easy access to Northern Europe, with possibility for rapid northward expansion, after ice retreat (Normand et al., 2011). While these species are indeed cold tolerant, their tolerance syndromes include some degree of drought tolerance as well, shifting them towards the center of the cold-drought trade-off axis. This effect has the potential to decouple these species from the negative relationship between the cold-drought trade-off and range size, indicating the importance of considering stress tolerance syndromes based on several factors rather than single tolerances when investigating interspecific differences in ranges. Other species that are potentially decoupled from this relationship belong to European genera that do not occur in the North American species set. More studies are needed for these European species. In the case of small range, drought-tolerant species, the explanation provided for North American species is likely to apply to European species as well (e.g. Normand et al., 2009).

The negative scaling of range size along the cold-drought tolerance trade-off is also consistent with macrophysiological evidence suggesting that pre-adaptation to low temperature or species-specific abilities to adapt to freezing temperatures may have favoured species' northward migration after glacial retreat (e.g. Araújo et al., 2013; Lancaster \& Humphreys, 2020). In contrast, it has been suggested that heat tolerances might be less adaptable (e.g. Araújo et al., 2013) and, considering that drought-tolerant species usually thrive in hot environments, 
adaptations to tolerate drought might have concomitantly prevented the northward post-glacial race for drought tolerant plants. This suggests that the cold-drought trade-off might limit niche expansion for temperate woody species, as has been suggested for heat stress globally (Araújo et al., 2013). Importantly, however, despite the fact that drought and heat stress can covary in warm-temperate ecosystems, we argue that the effect of drought tolerance on range size should be considered separately from that of heat tolerance. The most heat tolerant plants are from both dry and moist environments (Lancaster \& Humphreys 2020), and although transpirational cooling generally costs moisture, plants have also evolved adaptations to reduce the detrimental effects of heat stress under drought (Flexas et al., 2014). For example, among a plethora of other adaptations, changes in water-use efficiency (Flexas et al., 2014) or leaf movements (Puglielli et $a l ., 2017)$ can decouple leaf physiological responses to high temperatures from those to drought in Mediterranean woody plants.

We have shown that species positioning along the cold-drought trade-off axis imposes fundamental constraints upon interspecific differences in the range size of woody species, and contributes to shaping their realized niches (i.e. actual range) across continents. Species positioning in the Stress Space, which is determined by trade-offs between multiple tolerances (Puglielli et al., 2021a), can be interpreted as a measure of a species' realized niche based on abiotic resources (sensu Hutchinson, 1957) more than as a measure of the fundamental niche. After all the trade-off between tolerances shrinks fundamental niche size towards that of the realized niche (e.g. Sack, 2004). Therefore, by representing the typical multiple abiotic stress levels to which a plant species has adapted, its position in the Stress Space inherently corresponds to a set of energy constraints that define plant life history strategies. Such strategies are thought to directly control species' geographical distributions (Morin \& Chuine, 2006).

Niche position along resource-defined axes is a strong predictor of range size and occupancy in many animal groups (Seliger et al., 2021), and our results show that this is also true for temperate woody plant species.

\section{Range filling is not affected by the cold-drought tolerance trade-off}

All species are expected to have constraints on the extent to which they fill their range because of their specific physiological and ecological requirements (Paul et al., 2009), and their inherent trade-offs. Our results suggest that other factors that covary with latitude, not considered in this 
475

476

477

478

479

480

481

482

483

484

485

486

487

488

489

490

491

492

493

494

495

496

497

498

499

500

501

502

503

504

study (e.g. dispersal syndromes, Estrada et al., 2016), might be important for explaining range filling pattern. Such historical non-climatic limitations can ultimately also covary with species' abiotic stress tolerances. This might explain why a positive interaction between the cold-drought tolerance trade-off and latitude drives their range filling differences. Similarly, Nogués-Bravo et al. (2014) proposed that the negative correlation between seed mass and range filling found across 38 European tree species could be due to covariation between seed mass and other factors (e.g. drought tolerance), but further analysis including a larger number of species would be needed in order to test this claim.

More importantly, our results highlight different range filling determinants between the two continents, that contrarily to actual range, did not depend on differences in genera between continents (see Appendix S5, Table S5.4). European species' range filling was driven by a positive interaction between latitude and the cold-drought tolerance trade-off, while North American species' range filling was influenced only by a negative effect of latitude. This negative relationship between range filling and latitude for North American species was in agreement with a previous study that also reported a positive relationship range filling-longitude (Seliger et al., 2021), suggesting that longitude can indeed alter the expected range fillinglatitude relationship. Differences between continents might therefore depend on their geographical extent. Europe has a much smaller latitudinal and longitudinal range and less gradual geographical clines than North America (Morin \& Chuine, 2006). In addition, some European species ranges also occur outside Europe (e.g., species with a Eurasian distributions) and this might have influenced model's projections (see Appendix S3). According to the above, we therefore suggest that the interaction between these contrasting continental features and intrinsic drivers, such as dispersal syndromes, might determine continent-specific levels of range filling.

\section{Differences among plant functional types}

Plant functional type (PFTs) did not affect the negative scaling of range size with the colddrought trade-off (Table 1). However, the distribution of range size, range filling and positioning along the cold-drought trade-off axis differed between PFTs (Fig. 2 a-f). This probably explains why PFTs generally accounted for more of the random effects variance (even though the amount was generally low) for range metrics compared to phylogeny (Table 1). In general, despite some 
overlap between PFTs, deciduous angiosperms showed the highest values for both actual range and range filling (consistent with large-ranged species also having greater range filling, Seliger $e t$ al., 2021), and they were located further towards the cold tolerance end of the trade-off axis. Using a dataset of European and North American woody species, Morin \& Chuine (2006) also found that deciduous species were overrepresented among the large-ranged species.

Many deciduous angiosperms differ in trait syndromes from evergreen angiosperms or evergreen gymnosperms (e.g. Puglielli et al., 2021b), and different trait syndromes match differences in PFTs geographical distributions ( Zanne et al., 2018). For example, in the northern hemisphere, deciduous species tend to be more frequent in cold climates compared to evergreen broad-leaved angiosperms, despite some overlap between PFTs at almost all latitudes (Zanne $e t$ al., 2018). Conversely, while adaptations to tolerate drought closely match the distribution of evergreen species, this is not always true for deciduous species (e.g. see Kunert et al., 2021), suggesting that drought does not always limit deciduous species spatial distribution.

Deciduousness per se has been the common explanation for the ability of deciduous species to colonize either cold or dry environments, as it is an adaptation that permits avoidance of unfavorable environmental conditions. This explains why deciduous angiosperms in our dataset have larger ranges than other PFTs, and are mostly cold-tolerant. However, if deciduousness is also a successful drought avoidance strategy that could drive to large ranges in drought tolerant species as well, the relationship between range size and the trade-off axis should be not significant rather than negative. We can reconcile this by considering that woody species of the warm-temperate/temperate regions of the Northern Hemisphere are not generally droughtdeciduous. Drought tolerant deciduous species have sufficiently though leaves to widen their growing season beyond the usual duration of periods of drought (Hallik et al. 2009). Such adaptation is of course part of a wider trait syndrome (see Hallik et al., 2009), including, for example, greater biomass allocation to roots in species with greater drought-tolerance (Puglielli et al., 2021b), or smaller vascular conduits (Olson et al., 2018), that support the investment in tough leaves. This is consistent with the previous discussion of a link between species' tolerance strategies, trait syndromes and range size.

To summarize, while we recognize that adaptations to very low temperatures are complex, and involve a combination of avoidance and tolerance strategies together with 
acclimation (Schubert et al., 2020), we argue that large range sizes at the cold tolerant end of the trade-off axis is made viable because of the deciduous habit in temperate woody species. In this instance, stress avoidance is more important as an asset than stress tolerance. However, we also observed a greater overlap, in both range size and positioning along the trade-off axis, between freezing-tolerant North American gymnosperms and deciduous angiosperms than with evergreen angiosperms (Fig. 2 d,f). Thus, we do not exclude the additional role for freezing tolerance in guaranteeing large ranges.

\section{Conclusions}

Our results demonstrate that the cold-drought tolerance trade-off partly explains interspecific differences in range size across temperate woody plant species and that this relationship is largely independent of latitude and consistent with woody species biogeographical histories in the considered continents. Notably, our findings also suggest that accounting for species' abiotic stress tolerance towards multiple stresses can reconcile macroecological and macrophysiological theories aimed at explaining range size differences among woody plant species, supporting a previous hypothesis by Morin \& Chuine (2006). However, our results concerning the impact of abiotic stress tolerance on range filling were inconclusive, suggesting that other factors not studied here and that covary with latitude and/or abiotic stress tolerance syndromes are the main drivers of range filling in temperate woody species. Finally, our results also demonstrate the importance of considering trait syndromes, reflected here as differences between plant functional types, in clarifying the ways in which species' adaptations influence broad interspecific differences in range size in woody plant species in relation to abiotic stress tolerance.

\section{References}

Addo-Bediako, A., Chown, S.L. \& Gaston, K.J. (2000) Thermal tolerance, climatic variability and latitude. Proceedings of the Royal Society of London. Series B: Biological Sciences, 267, 739-745.

Allouche, O., Tsoar, A. \& Kadmon, R. (2006) Assessing the accuracy of species distribution models: prevalence, kappa and the true skill statistic (TSS). Journal of Applied Ecology, 43, 1223-1232. 
Araújo, M.B., Ferri $\square$ Yáñez, F., Bozinovic, F., Marquet, P.A., Valladares, F. \& Chown, S.L. (2013) Heat freezes niche evolution. Ecology Letters, 16, 1206-1219.

Brown, J.H. (1984) On the Relationship between Abundance and Distribution of Species. The American Naturalist, 124, 255-279.

Busby, J.R. (1986) A biogeoclimatic analysis of Nothofagus cunninghamii (Hook.) Oerst. in southeastern Australia. Australian Journal of Ecology, 11, 1-7.

Caudullo, G., Welk, E. \& San-Miguel-Ayanz, J. (2017) Chorological maps for the main European woody species. Data in Brief, 12, 662-666.

Chuine, I. (2010) Why does phenology drive species distribution? Philosophical Transactions of the Royal Society B: Biological Sciences, 365, 3149-3160.

Colwell, R.K. \& Hurtt, G.C. (1994) Nonbiological Gradients in Species Richness and a Spurious Rapoport Effect. The American Naturalist, 144, 570-595.

Estrada, A., Morales-Castilla, I., Caplat, P. \& Early, R. (2016) Usefulness of Species Traits in Predicting Range Shifts. Trends in Ecology \& Evolution, 31, 190-203.

Estrada, A., Morales $\square$ Castilla, I., Meireles, C., Caplat, P. \& Early, R. (2018) Equipped to cope with climate change: traits associated with range filling across European taxa. Ecography, 41, 770-781.

Fielding, A.H. \& Bell, J.F. (1997) A review of methods for the assessment of prediction errors in conservation presence/absence models. Environmental Conservation, 24, 38-49.

Fine, P.V.A. (2015) Ecological and Evolutionary Drivers of Geographic Variation in Species Diversity. Annual Review of Ecology, Evolution, and Systematics, 46, 369-392.

Flexas, J., Diaz-Espejo, A., Gago, J., Gallé, A., Galmés, J., Gulías, J. \& Medrano, H. (2014) Photosynthetic limitations in Mediterranean plants: A review. Environmental and Experimental Botany, 103, 12-23.

Freckleton, R.P., Harvey, P.H., Pagel, M. \& Losos, A.E.J.B. (2002) Phylogenetic Analysis and Comparative Data: A Test and Review of Evidence. The American Naturalist, 160, 712726.

Gaston, K.J., Chown, S.L., Calosi, P., Bernardo, J., Bilton, D.T., Clarke, A., Clusella $\square$ Trullas, S., Ghalambor, C.K., Konarzewski, M., Peck, L.S., Porter, W.P., Pörtner, H.O., Rezende, E.L., Schulte, P.M., Spicer, J.I., Stillman, J.H., Terblanche, J.S. \& Van Kleunen, M. 

(2009). Macrophysiology: A Conceptual Reunification. The American Naturalist, 174, 595-612.

Hijmans, R.J., Williams, E., Vennes, C. (2019). Spherical Trigonometry. R package available at https://CRAN.R-project.org/package=geosphere.

Grubb, P.J. (1977) The Maintenance of Species-Richness in Plant Communities: The Importance of the Regeneration Niche. Biological Reviews, 52, 107-145.

Guisan, A., Thuiller, W. \& Zimmermann, N.E. (2017) Habitat Suitability and Distribution Models: With Applications in R. Cambridge: Cambridge University Press.

Hallik, L., Niinemets, Ü. \& Wright, I.J. (2009) Are species shade and drought tolerance reflected in leaf-level structural and functional differentiation in Northern Hemisphere temperate woody flora? New Phytologist, 184, 257-274.

Hanski I, Kouki J, Halkka A. 1993. Three explanations of the positive relationship between distribution and abundance of species. In: Ricklefs R, Schluter D, eds. Species diversity in ecological communities: historical and geographical perspectives. Chicago, IL, USA: The University of Chicago Press, 108-116.

Hijmans, R.J., Cameron, S.E., Parra, J.L., Jones, P.G. \& Jarvis, A. (2005) Very high resolution interpolated climate surfaces for global land areas. International Journal of Climatology, 25, 1965-1978.

Hutchinson GE. 1957. Concluding Remarks. Cold Spring Harbor Symposia on Quantitative Biology 22: 415-427.

Ives, A.R. \& Helmus, M.R. (2011) Generalized linear mixed models for phylogenetic analyses of community structure. Ecological Monographs, 81, 511-525.

Jin, Y. \& Qian, H. (2019) V.PhyloMaker: an R package that can generate very large phylogenies for vascular plants. Ecography, 42, 1353-1359.

Kalwij, J.M., Robertson, M.P., Ronk, A., Zobel, M. \& Pärtel, M. (2014) Spatially-Explicit Estimation of Geographical Representation in Large-Scale Species Distribution Datasets. PLoS One, 9, e85306.

Koenker, R. 2017. Package 'quantreg.' R Found. Stat. Comp., Vienna, Austria.

Kunert, N., Zailaa, J., Herrmann, V., Muller-Landau, H.C., Wright, S.J., Pérez, R., McMahon, S.M., Condit, R.C., Hubbell, S.P., Sack, L., Davies, S.J. \& Anderson-Teixeira, K.J. 
(2021) Leaf turgor loss point shapes local and regional distributions of evergreen but not deciduous tropical trees. New Phytologist, 230, 485-496.

Laanisto, L. \& Niinemets, Ü. (2015) Polytolerance to abiotic stresses: how universal is the shade-drought tolerance trade-off in woody species? Global Ecology and Biogeography, 24, 571-580.

Lancaster, L.T. \& Humphreys, A.M. (2020) Global variation in the thermal tolerances of plants. Proceedings of the National Academy of Sciences USA, 117, 13580-13587.

Li, D., Dinnage, R., Nell, L.A., Helmus, M.R. \& Ives, A.R. (2020) phyr: An r package for phylogenetic species-distribution modelling in ecological communities. Methods in Ecology and Evolution, 11, 1455-1463.

Linder, H.P., Antonelli, A., Humphreys, A.M., Pirie, M.D. \& Wüest, R.O. (2013) What determines biogeographical ranges? Historical wanderings and ecological constraints in the danthonioid grasses. Journal of Biogeography, 40, 821-834.

Liu, C., White, M. \& Newell, G. (2013) Selecting thresholds for the prediction of species occurrence with presence-only data. Journal of Biogeography, 40, 778-789.

McFadden, I.R., Sandel, B., Tsirogiannis, C., Morueta-Holme, N., Svenning, J.-C., Enquist, B.J. \& Kraft, N.J.B. (2019) Temperature shapes opposing latitudinal gradients of plant taxonomic and phylogenetic $\beta$ diversity. Ecology Letters, 22, 1126-1135.

Morin, X. \& Chuine, I. (2006) Niche breadth, competitive strength and range size of tree species: a trade-off based framework to understand species distribution. Ecology Letters, 9, 185195.

Morueta $\square$ Holme, N., Enquist, B.J., McGill, B.J., Boyle, B., Jørgensen, P.M., Ott, J.E., Peet, R.K., Šímová, I., Sloat, L.L., Thiers, B., Violle, C., Wiser, S.K., Dolins, S., Donoghue, J.C., Kraft, N.J.B., Regetz, J., Schildhauer, M., Spencer, N. \& Svenning, J.-C. (2013) Habitat area and climate stability determine geographical variation in plant species range sizes. Ecology Letters, 16, 1446-1454.

Naimi, B. \& Araújo, M.B. (2016) sdm: a reproducible and extensible R platform for species distribution modelling. Ecography, 39, 368-375.

Niinemets, Ü. \& Valladares, F. (2006) Tolerance to Shade, Drought, and Waterlogging of Temperate Northern Hemisphere Trees and Shrubs. Ecological Monographs, 76, 521547. 
Nogués-Bravo, D., Pulido, F., Araújo, M.B., Diniz-Filho, J.A.F., García-Valdés, R., Kollmann, J., Svenning, J.-C., Valladares, F. \& Zavala, M.A. (2014) Phenotypic correlates of potential range size and range filling in European trees. Perspectives in Plant Ecology, Evolution and Systematics, 16, 219-227.

Normand, S., Ricklefs, R.E., Skov, F., Bladt, J., Tackenberg, O. \& Svenning, J.-C. (2011) Postglacial migration supplements climate in determining plant species ranges in Europe. Proceedings of the Royal Society B: Biological Sciences, 278, 3644-3653.

Normand, S., Treier, U.A., Randin, C., Vittoz, P., Guisan, A. \& Svenning, J.-C. (2009) Importance of abiotic stress as a range-limit determinant for European plants: insights from species responses to climatic gradients. Global Ecology and Biogeography, 18, 437-449.

Olson, M.E., Soriano, D., Rosell, J.A., Anfodillo, T., Donoghue, M.J., Edwards, E.J., LeónGómez, C., Dawson, T., Camarero Martínez, J.J., Castorena, M., Echeverría, A., Espinosa, C.I., Fajardo, A., Gazol, A., Isnard, S., Lima, R.S., Marcati, C.R. \& MéndezAlonzo, R. (2018) Plant height and hydraulic vulnerability to drought and cold. Proceedings of the National Academy of Sciences, 115, 7551-7556.

Paul, J.R., Morton, C., Taylor, C.M. \& Tonsor, S.J. (2009) Evolutionary Time for Dispersal Limits the Extent but Not the Occupancy of Species' Potential Ranges in the Tropical Plant Genus Psychotria (Rubiaceae). The American Naturalist, 173, 188-199.

Phillips, S.J., Anderson, R.P. \& Schapire, R.E. (2006) Maximum entropy modeling of species geographic distributions. Ecological Modelling, 190, 231-259.

Pither, J. (2003) Climate tolerance and interspecific variation in geographic range size. Proceedings of the Royal Society of London. Series B: Biological Sciences, 270, 475481.

Puglielli, G., Hutchings, M.J. \& Laanisto, L. (2021a) The triangular space of abiotic stress tolerance in woody species: a unified trade-off model. New Phytologist, 229, 1354-1362. Puglielli, G., Laanisto, L., Poorter, H. \& Niinemets, Ü. (2021b) Global patterns of biomass allocation in woody species with different tolerances of shade and drought: evidence for multiple strategies. New Phytologist, 229, 308-322. 
Puglielli, G., Redondo-Gómez, S., Gratani, L. \& Mateos-Naranjo, E. (2017) Highlighting the differential role of leaf paraheliotropism in two Mediterranean Cistus species under drought stress and well-watered conditions. Journal of Plant Physiology, 213, 199-208.

Ricotta, C., Godefroid, S. \& Rocchini, D. (2010) Patterns of native and exotic species richness in the urban flora of Brussels: rejecting the 'rich get richer' model. Biological Invasions, 12, $233-240$.

Sack, L. (2004) Responses of temperate woody seedlings to shade and drought: do trade-offs limit potential niche differentiation? Oikos, 107, 110-127.

Schubert, M., Humphreys, A.M., Lindberg, C.L., Preston, J.C. \& Fjellheim, S. (2020) To Coldly Go Where No Grass has Gone Before: A Multidisciplinary Review of Cold Adaptation in Poaceae. In Annual Plant Reviews online, J.A. Roberts (Ed.). https://doi.org/10.1002/9781119312994.apr0739.

Seliger, B.J., McGill, B.J., Svenning, J.-C. \& Gill, J.L. (2021) Widespread underfilling of the potential ranges of North American trees. Journal of Biogeography, 48, 359-371.

Sexton, J.P., Montiel, J., Shay, J.E., Stephens, M.R. \& Slatyer, R.A. (2017) Evolution of Ecological Niche Breadth. Annual Review of Ecology, Evolution, and Systematics, $\mathbf{4 8 ,}$ 183-206.

Sheth, S.N., Morueta $\square$ Holme, N. \& Angert, A.L. (2020) Determinants of geographic range size in plants. New Phytologist, 226, 650-665.

Smith, S.A. \& Brown, J.W. (2018) Constructing a broadly inclusive seed plant phylogeny. American Journal of Botany, 105, 302-314.

Stevens, G.C. (1989) The Latitudinal Gradient in Geographical Range: How so Many Species Coexist in the Tropics. The American Naturalist, 133, 240-256.

Sunday, J.M., Bates, A.E. \& Dulvy, N.K. (2011) Global analysis of thermal tolerance and latitude in ectotherms. Proceedings of the Royal Society B: Biological Sciences, 278, $1823-1830$.

Svenning, J.-C., Normand, S. \& Skov, F. (2008) Postglacial Dispersal Limitation of Widespread Forest Plant Species in Nemoral Europe. Ecography, 31, 316-326.

Svenning, J.-C. \& Skov, F. (2004) Limited filling of the potential range in European tree species. Ecology Letters, 7, 565-573. 
714

715

716

717

718

719

720

721

722

723

724

725

726

727

728

729

730

731

732

733

734

Title, P.O. \& Bemmels, J.B. (2018) ENVIREM: an expanded set of bioclimatic and topographic variables increases flexibility and improves performance of ecological niche modeling. Ecography, 41, 291-307.

Varela, S., Anderson, R.P., García $\square$ Valdés, R. \& Fernández $\square$ González, F. (2014)

Environmental filters reduce the effects of sampling bias and improve predictions of ecological niche models. Ecography, 37, 1084-1091.

Wazen, N., Garavaglia, V., Picard, N., Besacier, C. \& Fady, B. (2020) Distribution maps of twenty-four Mediterranean and European ecologically and economically important forest tree species compiled from historical data collections. Annals of Silvicultural Research, 44, 95-101.

Weiser, M.D., Enquist, B.J., Boyle, B., Killeen, T.J., Jørgensen, P.M., Fonseca, G., Jennings, M.D., Kerkhoff, A.J., Jr, T.E.L., Monteagudo, A., Vargas, M.P.N., Phillips, O.L., Swenson, N.G. \& Martínez, R.V. (2007) Latitudinal patterns of range size and species richness of New World woody plants. Global Ecology and Biogeography, 16, 679-688.

Zanne, A.E., Pearse, W.D., Cornwell, W.K., McGlinn, D.J., Wright, I.J. \& Uyeda, J.C. (2018) Functional biogeography of angiosperms: life at the extremes. New Phytologist, 218, $1697-1709$. 